\title{
Front Matter: Volume 10168
}

, "Front Matter: Volume 10168," Proc. SPIE 10168, Sensors and Smart Structures Technologies for Civil, Mechanical, and Aerospace Systems 2017, 1016801 (27 July 2017); doi: 10.1117/12.2280123

Event: SPIE Smart Structures and Materials + Nondestructive Evaluation and SPIE. Health Monitoring, 2017, Portland, Oregon, United States 


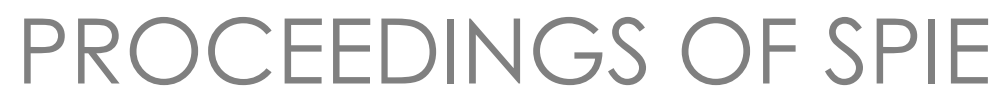

\section{Sensors and Smart Structures Technologies for Civil, Mechanical, and Aerospace Systems 2017}

Jerome P. Lynch

Editor

\section{6-29 March 2017 \\ Portland, Oregon, United States}

Sponsored by

SPIE

Co-sponsored by

OZ Optics, Ltd. (United States)

Polytec, Inc. (United States)

Fiberguide Industries (United States)

Frontiers Media (Switzerland)

Cooperating Organizations

Jet Propulsion Laboratory (United States)

Published by

SPIE

Part One of Two Parts

Volume 10168 
The papers in this volume were part of the technical conference cited on the cover and title page. Papers were selected and subject to review by the editors and conference program committee. Some conference presentations may not be available for publication. Additional papers and presentation recordings may be available online in the SPIE Digital Library at SPIEDigitallibrary.org.

The papers reflect the work and thoughts of the authors and are published herein as submitted. The publisher is not responsible for the validity of the information or for any outcomes resulting from reliance thereon.

Please use the following format to cite material from these proceedings:

Author(s), "Title of Paper," in Sensors and Smart Structures Technologies for Civil, Mechanical, and Aerospace Systems 2017, edited by Jerome P. Lynch, Proceedings of SPIE Vol. 10168 (SPIE, Bellingham, WA, 2017) Seven-digit Article CID Number.

ISSN: 0277-786X

ISSN: 1996-756X (electronic)

ISBN: 9781510608214

ISBN: 9781510608221 (electronic)

Published by

SPIE

P.O. Box 10, Bellingham, Washington 98227-0010 USA

Telephone +1 3606763290 (Pacific Time) · Fax +1 3606471445

SPIE.org

Copyright (c) 2017, Society of Photo-Optical Instrumentation Engineers.

Copying of material in this book for internal or personal use, or for the internal or personal use of specific clients, beyond the fair use provisions granted by the U.S. Copyright Law is authorized by SPIE subject to payment of copying fees. The Transactional Reporting Service base fee for this volume is $\$ 18.00$ per article (or portion thereof), which should be paid directly to the Copyright Clearance Center (CCC), 222 Rosewood Drive, Danvers, MA 01923. Payment may also be made electronically through CCC Online at copyright.com. Other copying for republication, resale, advertising or promotion, or any form of systematic or multiple reproduction of any material in this book is prohibited except with permission in writing from the publisher. The CCC fee code is $0277-786 \mathrm{X} / 17 / \$ 18.00$.

Printed in the United States of America.

Publication of record for individual papers is online in the SPIE Digital Library.

\section{SPIE. DIGITAL \\ SPIEDigitalLibrary.org}

Paper Numbering: Proceedings of SPIE follow an e-First publication model. A unique citation identifier (CID) number is assigned to each article at the time of publication. Utilization of CIDs allows articles to be fully citable as soon as they are published online, and connects the same identifier to all online and print versions of the publication. SPIE uses a seven-digit CID article numbering system structured as follows:

- The five digits correspond to the SPIE volume number.

- The last two digits indicate publication order within the volume using a Base 36 numbering

system employing both numerals and letters. These two-number sets start with 00, 01, 02, 03, 04, 05, 06, 07, 08, 09, OA, OB ... 0Z, followed by 10-1Z, 20-2Z, etc. The CID Number appears on each page of the manuscript. 


\title{
Contents
}

\author{
xi Authors \\ xV Conference Committee
}

\section{Part One}

ULTRASONIC INSPECTION METHODS FOR SHM

1016805 Effect of crack contact compression on the magnitude of nonlinear ultrasonic wave modulations [10168-3]

1016806 Multimodal location algorithm for Lamb waves propagating through anisotropic materials [10168-4]

1016807 Dispersion curve extraction of Lamb waves in metallic plates by matrix pencil method [10168-5]

1016808 The adhesive effect on ultrasonic Lamb wave detection sensitivity of remotely bonded fiber Bragg grating sensors [10168-6]

1016809 Damage identification in plate and shell structures by trilateration method using Lamb waves [10168-7]

10168 0A Synchronous separation, seaming, sealing and sterilization (\$4) using brazing for sample containerization and planetary protection [10168-135]

\section{THIN FILM SENSING SYSTEMS}

$10168 \mathrm{OB}$ A robust signal processing method for quantitative high-cycle fatigue crack monitoring using soft elastomeric capacitor sensors [10168-8]

$10168 \mathrm{OC}$ On the modeling and characterization of an interlocked flexible electronic skin [10168-9]

$10168 \mathrm{OE}$ 3D printing of highly elastic strain sensors using polyurethane/multiwall carbon nanotube composites [10168-11]

10168 OF Enhanced PVDF properties by multi-wall carbon nanotubes (MWCNT) for efficient energy harvesting [10168-12]

10168 OG Development of self-powered strain sensor using mechano-luminescent ZnS:Cu and mechano-optoelectronic P3HT [10168-13]

$10168 \mathrm{OH}$ Semiconductor nanomembrane-based sensors for high frequency pressure measurements [10168-14] 
$10168 \mathrm{OJ}$ Shape information model of large structure using terrestrial laser scanning [10168-16]

$10168 \mathrm{OL} \quad$ Visual based laser speckle pattern recognition method for structural health monitoring [10168-18]

MONITORING STRATEGIES FOR CIVIL INFRASTRUCTURE

$101680 \mathrm{M} \quad$ Urban underground infrastructure mapping and assessment [10168-19]

1016800 Recursive subspace-based identification of linear time-varying system [10168-21]

$10168 \mathrm{OP} \quad$ Evaluation of truss bridges using distributed strain measurements [10168-22]

$101680 Q \quad$ A new method for detection of fatigue cracking in steel bridge girders using self-powered wireless sensors [10168-23]

\section{NOVEL SENSING TECHNOLOGIES I}

$10168 \mathrm{OR}$ Heating and thermal control of brazing technique to break contamination path for potential Mars sample return [10168-24]

10168 OS Variable input observer for state estimation of high-rate dynamics [10168-25]

10168 OT Accelerated damage visualization using binary search with fixed distance laser ultrasonic scanning [10168-26]

$10168 \mathrm{OU}$ Physics-based structural health monitoring using the time-frequency analysis of electromechanical impedance signals [10168-27]

$101680 \mathrm{~V}$ Strain field reconstruction on composite spars based on the identification of equivalent load conditions [10168-28]

$10168 \mathrm{OW}$ Inverse analysis of aerodynamic loads from strain information using structural models and neural networks [10168-29]

$101680 X \quad$ A machine-learning approach for damage detection in aircraft structures using selfpowered sensor data [10168-30]

\section{STRUCTURAL HEALTH MONITORING FOR STRUCTURAL AND GEOTECHNICAL SYSTEMS}

10168 OY A probabilistic model for visual inspection of concrete shear walls [10168-31]

$101680 Z$ Performance assessment of geotechnical structural elements using distributed fiber optic sensing [10168-32] 
1016810 Response reduction methods for base isolated buildings with collision to retaining walls [10168-33]

1016811 Damage detection of structures by wavelet analysis: application to seismic response of steel frames [10168-34]

1016812 Detecting subsurface features and distresses of roadways and bridge decks with ground penetrating radar at traffic speed [10168-140]

MULTIFUNCTIONAL MATERIALS FOR STRUCTURAL HEALTH MONITORING

1016813 Damage detection and localization algorithm using a dense sensor network of thin film sensors [10168-36]

1016815 Experimental damage detection of wind turbine blade using thin film sensor array [10168-38]

1016816 Simultaneous sensing of fluid velocity and temperature using particle tracers embedding nitrobenzofurazan functionalized thermosensitive hydrogels [10168-39]

1016817 Dynamic piezoresistive response of hybrid nanocomposites [10168-40]

\section{SENSING HUMAN OCCUPANTS IN BUILDINGS}

1016818 Design and development of a prototype platform for gait analysis [10168-41]

1016819 Characterizing left-right gait balance using footstep-induced structural vibrations [10168-42]

$101681 \mathrm{~A} \quad$ Classification of event location using matched filters via on-floor accelerometers [10168-43]

10168 1B Design of smart prosthetic knee utilizing magnetorheological damper [10168-44] NOVEL SENSING TECHNOLOGIES III

$101681 \mathrm{C}$ Structural analysis and aeroservoelastic response simulation of smart fin operated by piezoelectric actuators [10168-45]

10168 1D Determination of orthotropic mechanical properties of 3D printed parts for structural health monitoring [10168-46]

$101681 \mathrm{E} \quad$ Finite difference analysis and experimental validation of 3D photonic crystals for structural health monitoring [10168-47]

10168 1F Improved equivalent circuit modeling and simulation of magnetostrictive tuning fork gyro sensors [10168-48] 
$101681 \mathrm{G}$ Measurements of multicore microstructured optical fibers heated up to $50^{\circ} \mathrm{C}$ [10168-49]

$101681 \mathrm{H} \quad$ A study on MFL based wire rope damage detection [10168-50]

$101681 \mathrm{~J} \quad$ A spectral profile multiplexed FBG sensor network with application to strain measurement in a Kevlar woven fabric [10168-52]

$101681 \mathrm{~K} \quad$ Noncontact tomography and a pH-sensitive nanocomposite for monitoring osseointegrated prosthesis interfaces [10168-53]

$101681 \mathrm{~L} \quad$ Fiber gratings strain sensor systems for composites and adhesive joints [10168-54]

$101681 \mathrm{M} \quad$ Feasibility of magnetic fiber-optic based corrosion sensor [10168-55]

$101681 \mathrm{~N}$ Optical sensing of metal tiny particles using ceria nanoparticles via fluorescence quenching technique [10168-56]

\section{Part Two}

FIBER OPTIC SENSORS FOR STRUCTURAL HEALTH MONITORING

$101681 \mathrm{P} \quad$ Shape sensing of inflatable aerospace structures with fiber optic curvature rosettes [10168-58]

$101681 Q \quad$ Test of FBG sensors for monitoring high pressure pipes [10168-59]

10168 1R Corrosion detection for steel with soft coating using in-line fiber Bragg grating sensor [10168-60]

10168 is Distributed fiber optic strain sensing to detect artificial pitting corrosion in stirrups [10168-61]

$101681 \mathrm{~A} \quad$ A buoyancy-based fiber Bragg grating tilt sensor [10168-62]

$101681 \mathrm{U} \quad$ Embedded fiber Bragg grating sensor in composite for bandwidth modulation [10168-63]

$101681 \mathrm{~V} \quad$ Effect of gauge length on embedded fibre Bragg grating sensor response in woven fibre composites [10168-64]

\section{MONITORING STRATEGIES FOR BRIDGES}

$101681 \mathrm{X} \quad$ Utilization of wireless structural health monitoring as decision making tools for a condition and reliability-based assessment of railroad bridges [10168-66]

$101681 Y \quad$ In-pavement fiber Bragg grating sensors for high-speed weigh-in-motion measurements [10168-67] 
1016812 Investigating extreme event loading on coastal bridges using wireless sensor technology [10168-68]

1016820 Smart photonic coating for civil engineering field: for a future inspection technology on concrete bridge [10168-69]

$1016821 \quad$ Simultaneous identification method of damage and vehicle parameters on bridges utilizing long-gauge strain influence line under moving vehicle loads [10168-70]

\section{ENERGY SAVING AND HARVESTING METHODS FOR SENSORS}

1016822 Effectiveness of compressed sensing and transmission in wireless sensor networks for structural health monitoring [10168-100]

1016823 Compact piezoelectric resonance mass balance for sample verification and mass quantification and mixing [10168-71]

1016824 Energy harvesting from acoustic fields for self-powered sensors in pumped fluid systems [10168-72]

1016825 Design and experimental studies of an energy harvesting backpack with mechanical motion rectification [10168-73]

\section{ULTRASONIC METHODS}

1016827 Impedance-based structural health monitoring of additive manufactured structures with embedded piezoelectric wafers [10168-75]

1016828 Damage imaging of an isotropic plate using matching pursuit algorithm [10168-76]

1016829 Ultrasonic longitudinal waves to monitor the integration of titanium rods with host bone [10168-77]

10168 2A Sensor network performance and reliability evaluation algorithms [10168-78]

\section{CONTROL OF LARGE-SCALE STRUCTURES}

10168 2D Adaptive vibration control of structures under earthquakes [10168-80]

$101682 \mathrm{E} \quad$ A new self-powered electromagnetic damper for structural vibration control [10168-81]

$101682 \mathrm{~F} \quad$ Integrated cable vibration control system using wireless sensors [10168-82]

$101682 \mathrm{G}$ Characteristics of spaceborne cooler vibration isolator using a pseudoelastic shape memory alloy [10168-83] 
$101682 \mathrm{H} \quad$ Operational modal analysis of a steel-frame, low-rise building with L-shaped construction [10168-84]

$1016821 \quad$ Sparse reconstruction localization of multiple acoustic emissions in large diameter pipelines [10168-85]

10168 2K Detection of surface cracking in steel pipes based on vibration data using a multi-class support vector machine classifier [10168-87]

$101682 \mathrm{~L}$ Detection and assessment of flaws in friction stir welded metallic plates [10168-88]

\section{NOVEL SENSING TECHNOLOGIES IV}

$101682 \mathrm{~N}$ Structural health monitoring using a hybrid network of self-powered accelerometer and strain sensors [10168-90]

$101682 \mathrm{P} \quad$ Tunable mechanical monolithic sensors for real-time broadband distributed monitoring of large civil and industrial infrastructures [10168-92]

$101682 \mathrm{E}$ Estimation of the strength development of ultra high performance concrete using a single embedded sensor [10168-93]

$1016825 \quad$ Measurement of greenhouse gases in UAE by using Unmanned Aerial Vehicle (UAV) [10168-95]

\section{SYSTEM IDENTIFICATION AND DAMAGE DETECTION METHODS}

$101682 \mathrm{~T} \quad$ Stochastic subspace system identification using multivariate time-frequency distributions [10168-97]

$101682 \mathrm{U}$ Structural damage detection using high dimension data reduction and visualization techniques [10168-98]

$101682 \mathrm{~V}$ Vibration analysis of discrete parameter systems using fractional order models [10168-99]

10168 2W A distributed cloud-based cyberinfrastructure framework for integrated bridge monitoring [10168-139]

\section{POSTER SESSION}

1016830 Direct laser writing of polymer micro-ring resonator ultrasonic sensors [10168-104]

1016831 Classification of composite damage from FBG load monitoring signals [10168-105]

1016832 Control of equipment isolation system using wavelet-based hybrid sliding mode control [10168-106] 
1016835 Development of a slip sensor using separable bilayer with Ecoflex-NBR film [10168-109]

1016836 Design and analysis of compound flexible skin based on deformable honeycomb [10168-110]

1016837 Design of flexible skin based on a mixed cruciform honeycomb [10168-111]

1016838 Vibration characteristics of an ultrasonic transducer composed of inner and outer discs [10168-112]

1016839 Design and validation of wireless system for oil monitoring base on optical sensing unit [10168-113]

10168 3A Design of wireless synchronous structural monitoring system for large bridges [10168-114]

10168 3B Research on high-speed railway's vibration analysis checking based on intelligent mobile terminal [10168-115]

10168 3C Research on the ride comfort of elevator monitoring using smartphone [10168-116]

10168 3E Macro-fiber composites under thermal cycles for space applications [10168-118]

10168 3F Active mass damper system for high-rise buildings using neural oscillator and position controller: sinusoidally varying desired displacement of auxiliary mass intended for reduction of maximum control force [10168-119]

$101683 G$ Development of sensing systems printed with conductive ink on gear surfaces: manufacturing of meander line antenna by laser-sintered silver nano-particles [10168-120]

$101683 \mathrm{H} \quad$ Optimal control of build height utilizing optical profilometry in cold spray deposits [10168-121]

10168 3J Reliability-based optimization of an active vibration controller using evolutionary algorithms [10168-123]

10168 3K Metal rubber sensor technology to enable in-flight icing measurement [10168-124]

10168 3L Low frequency motion measurement of spacecrafts and satellites with inertial monolithic sensors [10168-125]

1016830 A magnetostrictive phased array sensor using a nickel comb patch for guided Lamb wave-based damage detection [10168-128]

10168 3P Prediction-error variance in Bayesian model updating: a comparative study [10168-129]

$101683 Q \quad$ Vibration measurement on composite material with embedded optical fiber based on phase-OTDR [10168-130]

$101683 R \quad$ Evaluation of a new source localization method in a simulated dispersive plate [10168-131]

1016835 Inversion-based imaging using lagrange polynomial parameterization and genetic algorithm optimization [10168-132] 
Proc. of SPIE Vol. 10168 1016801-10 Downloaded From: https://www.spiedigitallibrary.org/conference-proceedings-of-spie on 26 Apr 2023
Terms of Use: https://www.spiedigitallibrary.org/terms-of-use 


\section{Authors}

Numbers in the index correspond to the last two digits of the seven-digit citation identifier (CID) article numbering system used in Proceedings of SPIE. The first five digits reflect the volume number. Base 36 numbering is employed for the last two digits and indicates the order of articles within the volume. Numbers start with $00,01,02,03,04,05,06,07,08,09,0 A, 0 B . .0 Z$, followed by 10-1Z, 20-2Z, etc.

Abdelrhman, Mohammed, $2 S$

Abou-Elnour, Ali, $2 S$

Acernese, F., 3L

Acosta, Krystal L., 3E

Airoldi, A., OV

Alajlouni, Sa'ed, 1A, 3R

Alavi, Amir H., OQ, 2N

Aliheidari, Nahal, OE

Almahmoud, Safieh, IM

Al-Tarawneh, Mu'ath, IY

Ameli, Amir, OE

Amira, Abdelraouf, $2 S$

Anees, Muhammad, 17

Anton, Steven R., 1D, 27

Apicella, A., OV

Arun P., Durai, 09, 2A

Asadollahi, Parisa, 3P

Asanuma, Hiroshi, 1Q

Ayoub, Georges, 2L

Azab, M., $1 \mathrm{~N}$

Azarmi, Fardad, IR

Badescu, Mircea, OA, OR

Bales, D. B., 18

Balkis, Ahmed, $2 S$

Bao, Xiaoqi, OA, OR

Bar-Cohen, Yoseph, OA, OR

Barone, F., 2P, 3L

Bennett, Caroline, OB

Berg, Michelle, 3K

Bettini, P., OV

Birken, Ralf, 12

Birnkrant, Michael, 3H

Biswas, Subir, OX

Bolander, John E., $2 Q$

Bond, Justin M., IP

Bradford, Philip D., 08

Braytee, A., 2K

Brown, Fredericka, $1 \mathrm{G}$

Bui, Viet Phuong, 3S

Burgueño, Rigoberto, OX

C. N., Sathyanarayana, 09, 2A

Calà Lesina, Antonino, $1 \mathrm{E}$

Campos, Sergio, OA, OR

Cao, Liang, OS

Cellini, Filippo, 16

Cha, Gichun, 0J

Chakrabartty, Shantanu, OX

Chakraborty, Abhijit, 3H

Chang, C. Y., 07
Chang, Chia-Ming, 2T

Chang, Chih Chen, 2E

Chaturvedi, Tanmay, IT

Chen, Jun-Da, 00

Chen, Shizhi, 21

Chiappini, Andrea, $1 \mathrm{E}$

Cho, Soojin, 2F

Choi, Hyouk Ryeol, 35

Christ, Josef F., OE

Claus, Richard O., OH, 3K

Collins, William, OB

Colucci, Alessandro, 1Q

Cunefare, Kenneth A., 24

Das, Saptarshi, OX

Davis, Claire, $1 \mathrm{~V}$

Davis, Justin R., $1 Z$

De Canio, Gerardo, $1 Q$

Deng, Fodan, $1 \mathrm{R}$

Deseri, Luca, $1 \mathrm{E}$

Diffenbaugh, T. E., 18

Dodson, Jacob, OS

Downey, Austin, 13, 15

Dubuc, Brennan, 2

Ebrahimkhanlou, Arvin, OY, 2

Erturk, Alper, 24

Ettouney, Mohammed, $1 \mathrm{X}$

Fagert, Jonathon, 19

Fakih, Mohammad Ali, 2L

Fan, Taian, OM

Felli, Ferdinando, 1Q

Ferrari, Maurizio, $1 \mathrm{E}$

Flanigan, Katherine A., IX

Flatau, A. B., 1F

Floridia, C., $3 Q$

Franciscangelis, C., 3Q

Freeman, David, OA

Fruett, F., 3Q

Fudouzi, Hiroshi, 20

Fujitani, Hideo, 10

Fujiwara, Takahiro, 22

Futagawa, S., $3 G$

Gaballah, S., 1N

Gao, F., 1B

Garcia, V., 18

Gbaguidi, Audrey, 17

Gelineau, Douglas A., $1 Z$

Giordano, G., 2P, 3L

Groves, Roger M., 31

Guo, Guodong, $1 \mathrm{~J}$ 
Gupta, Sumit, 1K

Hackney, Drew A., 08, $1 \mathrm{~J}$

Hallander, P., 3Q

Hällstrom, S., 3Q

Hamade, Ramsey, 2L

Han, Ming, $1 U$

Hasni, Hassene, $0 Q, 2 N$

Hayden, Martin, $\mathrm{OZ}$

Hendriks, Richard C., 31

Heusdens, Richard, 31

Hisel, Jacob, $1 G$

Hobeck, Jared D., 3E

Hohimer, Cameron J., OE

Hollkamp, John P., 2V

Homer, Michelle, $\mathrm{OH}$

Hong, Jonathan, OS

Hong, Jung-Wuk, 05

Hongu, J., 3F

Hoover, George, OG

Hor, Yew Li, $3 S$

Hou, Rui, 1X, 2W

Hoult, Neil A., OP, IS

Hsueh, Wen, 11

$\mathrm{Hu}$, Jie, OF

Huang, Haiying, OU, $1 \mathrm{U}$

Huang, Hui, 39

Huang, Shieh-Kung, 2T, 32

Huang, Ying, $1 R, 1 Y$

Huang, Yong, 3P

Huston, Dryver R., OM, IP

Hyakutake, Tsuyoshi, 20

Iba, D., 3F, 3G

lizuka, T., 3G

lliff, G. J., 18

Im, Byeong Uk, $1 \mathrm{C}$

Inman, Daniel J., 3E

Jagani, J., 18

Jamshidi, Maziar, 2E

Jeong, Seongwoon, $2 \mathrm{~W}$

Jeong, Seunghoo, 2F

Jiao, Pengcheng, $0 Q, 2 \mathrm{~N}$

Jin, Suyeong, 05

Jo, Hongki, OB

Johnson, Nephi R., $1 \mathrm{X}$

Jones, Tyler, $\mathrm{OH}$

Juang, Jer-Nan, 2D

Kamimoto, T., 3G

Kancharla, Vinutha, is

Kang, Yoo-Jin, 1C

Kang, Yuhong, $\mathrm{OH}$

Kasarda, Mary E., 2H

Kawai, Hiroyuki, 22

Khalili, Nazanin, OC

Kim, Daewon, 17

Kim, H. W., 28

Kim, J., 1H

Kim, J.-W., 1H

Kim, Jin O., 38

Kim, Sung Joon, 35

Kishida, Akiko, 10
Kong, Xiangxiong, $\mathrm{OB}$

Koo, Ja Choon, 35

Kreeger, Richard E., 3K

Krishnaswamy, Sridhar, 30

Kwon, Seong-Cheol, 2G

Laflamme, Simon, OB, OS, 13, 15

Lajnef, Nizar, $0 Q, 2 N$

Lalli, Jennifer, 3K

Lascelles, Alyson J., OP

Law, Kincho H., 2W

Lee, Changgil, $2 Q$

Lee, Donghwan, OJ

Lee, Hyeong Jae, 0A, 23

Lee, Sang Eon, 05

Lew, Jiann-Shiun, 2D

$\mathrm{Li}$, Jian, OB, 3P

Li, Na, 3A

Li, Peigang, 3B

Li, Zhirui, 39

Liao, W. H., 1B

Lienhart, Werner, $\mathrm{OZ}$

Lin, Jia-Hua, $2 \mathrm{U}$

Liu, Hao, 12

Liu, Mingyi, 25

Liu, Wei, 3A

Liv, Y. N., 1B

Liu, Zhiqiang, 3A

Loh, Chin-Hsiung, 0O, 11, 2D, 2U, 32

Loh, Kenneth J., IK

Lynch, Jerome P., 1X, 29, 2W

Maheshwari, Muneesh, IT

Malladi, V. V. N. Sriram, 18, 1A

Mao, Xingquan, $3 \mathrm{~A}$

Marelli, L., OV

Margulis, W., 3Q

Marschner, U., IF

Marti, M. A., 18

Masuda, A., 3G

Mayo, David, $\mathrm{OH}$

Merewether, Gene B., 23

Mirshekari, Mostafa, 19

Miura, N., 3G

Mo, Changki, OE

Mokhtar, B., IN

Mongelli, Marialuisa, 1Q

Monsberger, Christoph, $\mathrm{OZ}$

Moon, Hyungpil, 35

Moriwaki, I., 3F, 3G

Mukai, Yoichi, 10

Mustapha, Samir, 2K, 2L

Naguib, Hani E., OC

Nakamura, M., 3F, 3G

Namilae, Sirish, 17

$\mathrm{Ng}$, Wing, $\mathrm{OH}$

Nishimura, Nao, 10

Nishizaki, Itaru, 20

Nitta, Hiroyuki, 20

Niu, Liqun, 39

Noell, Aaron C., 23

Noh, Hae Young, 19 
Norman, Patrick, IV

Nyman, T., 3Q

Odeh, Mohamed, $2 S$

Oh, Hyun-Ung, 2G

Ohya, Takao, 20

Orfeo, Dan, OM

Owen, Robert B., 3E

Paget, Christophe A., 06

Pan, Shijia, 19

Pankow, Mark, $1 \mathrm{~J}$

Paolozzi, Antonio, 1Q

Paris, Claudio, 1Q

Park, Byeongjin, OT

Park, Chul-Woo, $1 \mathrm{C}$

Park, J., 1H

Park, Ji-Hwan, OJ

Park, Kyeongtaek, OL

Park, Seunghee, OJ, 1H, 2Q

Park, Yeon-Hyeok, 2G

Perry, Marcus, 1E

Peters, Kara J., 08, $1 \mathrm{~J}$

Peterson, Sean D., 16

Petersson, M., 3Q

Philips, Andrew, 1V

Phoenix, A., 18

Piao, Chunguang, 38

Piccolo, Valentina, $1 \mathrm{E}$

Pines, Darryll J., 30

Png, Ching Eng, 3S

Poissenot-Arrigoni, Bastien, 1D

Porfiri, Maurizio, 16

Pötschke, Petra, OE

Pourtakdoust, Seid H., 3J

Pulliam, Elias, OG

Pyo, Sukhoon, $2 Q$

Račanský, Václav, $0 z$

Rajabzadeh, Aydin, 31

Rajic, Nik, IV

Razinger, Jonathan, $\mathrm{OM}$

Rehman, M. Abdul, 06

Rice, Jennifer A., $1 Z$

Rizk, M. R. M., IN

Romano, R., 3L

Rong, Jiaxin, 37

Rosolem, J. B., 3Q

Rostron, Paul, $1 \mathrm{M}$

Ruan, Hang, $\mathrm{OH}$

Ryu, Donghyeon, OG

S., Raja, 09, 2A

Sala, G., OV

Salamone, Salvatore, OY, 2

Salehi, Hadi, OX

Salgado, F. C., 3Q

Samir, E., IN

Saraygord Afshari, Sajad, 3J

Sarkar, Partha, 15

Sarlo, Rodrigo, $2 \mathrm{H}$

Sasaki, T., 3F

Scheel, Ingrid, $1 \mathrm{~L}$

Scheyer, Austin G., 1D, 27
Schwartz, Forest J., 24

Semperlotti, Fabio, 2V

Sen, Mihir, $2 \mathrm{~V}$

Shehata, N., iN

Shen, Xuechen, OC

Sherrit, Stewart, OA, OR, 23

Shima, S., 3F

Shin, SangJoon, 1C

Shiryayev, Oleg, 1M

Shishkin, Sergey, 3H

Sibold, Ridge, $\mathrm{OH}$

Sim, Sung-Han, $2 F$

Singh, Abhay K., $1 \mathrm{U}$

Skow, Ellen A., 24

Söderquist, I., 3Q

Sohn, Hoon, OT, 2W

Sone, A., 3G

Starke, E., IF

Su, Wensheng, 3C

Sugimoto, Yohei, oW

Sun, Xiaowei, 3C

Taga, Kenzo, 10

Tai, Wei-Che, 25

Tanaka, Yoshikazu, 20

Tarazaga, Pablo A., 18, 1A, 2H, 3R

Tarraf, Jaafar, 2L

Tiparti, Dhruv, OG

Todoroki, Shin-ichi, 20

Tokairin, Tomoya, 22

Torbol, Marco, OL

Tsuchiya, Koichi, 20

Ubertini, Filippo, 13, 15

Uchiito, Haruki, 22

Udd, Eric, 1L

V.P. S., Naidu, 09, 2A

Vaccari, Alessandro, 1E

Vahdati, Nader, $1 \mathrm{M}$

Van Der Kooi, Kyle E., OP

Vendittozzi, Cristian, 1Q

Wada, Daichi, OW

Wagner, Gerald, IG

Wang, Ming L., 12

Wang, Weiming, 39

Wang, Wentao, 29

Wee, Junghyun, 08

Wei, Heming, 30

Woolard, Americo G., 18, 1A, 3R

Woschitz, Helmut, $\mathrm{OZ}$

Wu, Bitao, 21

Wu, Gang, 21

Wynne, Rosalind, $1 G$

Xia, Tian, OM

Xie, Shulin, 3B

Xie, Zhao, 3C

Xue, Zhigang, 3C

Yahnker, Christopher R., 23

Yamashita, Yuki, 10

Yang, Yaowen, IT

Yang, Zhitao, 3A

Ye, L., 2K 
Yoo, Byungseok, 30

Yoo, J.-H., IF

Yoon, Bum Soo, $1 \mathrm{C}$

Yoon, Kwang Joon, $1 \mathrm{C}$

Yu, ByoungJoon, OJ

Yu, Tzuyang, OF

Yu, Yan, 39, 3A

Yuan, F. G., 07, 28

Yuan, Yue, 25

Zahedi, Farshad, OU

Zhang, Jiachen, is

Zhang, Pei, 19

Zhang, Shuaishuai, 39

Zhang, Yang, 3C

Zhang, Yu, OM

Zhao, Huapeng, 3S

Zhao, Xuefeng, 3B, 3C

Zhong, Yu, 3S

Zhou, Li, 36, 37

Zhu, Yupeng, $1 \mathrm{U}$

Zonta, Daniele, $1 \mathrm{E}$

Zou, Tingting, 36

Zuo, Lei, 25 


\title{
Conference Committee
}

\author{
Symposium Chairs
}

Jayanth N. Kudva, NextGen Aeronautics, Inc. (United States)

Theodoros E. Matikas, University of loannina (Greece)

Symposium Co-chairs

Tribikram Kundu, The University of Arizona (United States)

Gregory W. Reich, Air Force Research Laboratory (United States)

Conference Chair

Jerome P. Lynch, University of Michigan (United States)

Conference Co-chairs

Hoon Sohn, KAIST (Korea, Republic of)

Kon-Well Wang, University of Michigan (United States)

Conference Program Committee

Hiroshi Asanuma, Chiba University (Japan)

Xiaoyi Bao, University of Ottawa (Canada)

Chih Chen Chang, Hong Kong University of Science and Technology

(Hong Kong, China)

Genda Chen, Missouri University of Science and Technology

(United States)

Wolfgang Ecke, Leibniz-Institut für Photonische Technologien e.V.

(Germany)

Alison B. Flatau, University of Maryland, College Park (United States)

Branko Glisic, Princeton University (United States)

Faramarz Gordaninejad, University of Nevada, Reno (United States)

Benjamin K. Henderson, Air Force Research Laboratory

(United States)

Jung-Wuk Hong, KAIST (Korea, Republic of)

Neil A. Hoult, Queen's University (Canada)

Haiying Huang, The University of Texas at Arlington (United States)

Ying Huang, North Dakota State University (United States)

Shinae Jang, University of Connecticut (United States)

Jeong-Tae Kim, Pukyong National University (Korea, Republic of)

Junhee Kim, Dankook University (Korea, Republic of)

Masahiro Kurata, Kyoto University (Japan)

Simon Laflamme, lowa State University (United States) 
Francesco Lanza di Scalea, University of California, San Diego (United States)

Hui Li, Harbin Institute of Technology (China)

Wei-Hsin Liao, The Chinese University of Hong Kong (Hong Kong, China)

Chin-Hsiung Loh, National Taiwan University (Taiwan)

Kenneth J. Loh, University of California, San Diego (United States)

Bryan R. Loyola, Sandia National Laboratories (United States)

David Dennis Lee Mascarenas, Los Alamos National Laboratory

(United States)

Theodore E. Matikas, University of loannina (Greece)

Norbert G. Meyendorf, lowa State University of Science and Technology (United States)

Akira Mita, Keio University (Japan)

Tomonori Nagayama, The University of Tokyo (Japan)

Yiqing Ni, The Hong Kong Polytechnic University (Hong Kong, China)

Hae Young Noh, Carnegie Mellon University (United States)

Irving J. Oppenheim, Carnegie Mellon University (United States)

Wieslaw M. Ostachowicz, The Szewalski Institute of Fluid-Flow

Machinery (Poland)

Jinping Ou, Dalian University of Technology (China)

Shamim N. Pakzad, Lehigh University (United States)

Seunghee Park, Sungkyunkwan University (Korea, Republic of)

Kara J. Peters, North Carolina State University (United States)

Michael K. Philen, Virginia Polytechnic Institute and State University

(United States)

Paul Reynolds, University of Exeter (United Kingdom)

Massimo Ruzzene, Georgia Institute of Technology (United States)

Liming W. Salvino, Office of Naval Research Global (United States)

Jeffrey T. Scruggs, University of Michigan (United States)

Fabio Semperlotti, Purdue University (United States)

Sung-Han Sim, Ulsan National Institute of Science and Technology

(Korea, Republic of)

Wei Song, The University of Alabama (United States)

Billie F. Spencer Jr., University of Illinois at Urbana-Champaign

(United States)

Wieslaw J. Staszewski, AGH University of Science and Technology

(Poland)

R. Andrew Swartz, Michigan Technological University (United States)

Michael D. Todd, University of California, San Diego (United States)

Masayoshi Tomizuka, University of California, Berkeley (United States)

Ming L. Wang, Northeastern University (United States)

Xingwei Wang, University of Massachusetts Lowell (United States)

Yang Wang, Georgia Institute of Technology (United States)

Rosalind M. Wynne, Villanova University (United States)

Fuh-Gwo Yuan, North Carolina State University (United States)

Chung-Bang Yun, Ulsan National Institute of Science and Technology

(Korea, Republic of) 
Yunfeng Zhang, University of Maryland, College Park (United States)

Daniele Zonta, University degli Studi di Trento (Italy)

\section{Session Chairs}

1 Keynote Session

Hoon Sohn, KAIST (Korea, Republic of)

Jerome P. Lynch, University of Michigan (United States)

2 Ultrasonic Inspection Methods for SHM

Jung-Wuk Hong, KAIST (Korea, Republic of)

Kara Peters, North Carolina State University (United States)

3 Thin Film Sensing Systems

Donghyeon Ryu, New Mexico Institute of Mining and Technology (United States)

Kenneth J. Loh, University of California, San Diego (United States)

4 Vision-based Sensing for Structural Health Monitoring

Marco Torbol, Ulsan National Institute of Science and Technology (Korea, Republic of)

5 Monitoring Strategies for Civil Infrastructure

Dryver R. Huston, The University of Vermont (United States)

Neil Hoult, Queen's University (Canada)

6 Novel Sensing Technologies I

Hoon Sohn, KAIST (Korea, Republic of)

Donghyeon Ryu, New Mexico Institute of Mining and Technology (United States)

7A Structural Health Monitoring for Structural and Geotechnical Systems Yongchao Yang, Los Alamos National Laboratory (United States)

7B Multifunctional Materials for Structural Health Monitoring

Simon Laflamme, lowa State University College of Engineering (United States)

Maurizio Porfiri, NYU Tandon School of Engineering (United States)

8A Sensing Human Occupants in Buildings

Hae Young Noh, Carnegie Mellon University (United States)

Fuh-Gwo Yuan, North Carolina State University (United States)

8B Novel Sensing Technologies III

Daniele Zonta, Università degli Studi di Trento (Italy)

Haiying Huang, The University of Texas at Arlington (United States) 
9A Novel Sensing Technologies II

Kenneth J. Loh, University of California, San Diego (United States)

Rosalind M. Wynne, Villanova University (United States)

9B Fiber Optic Sensors for Structural Health Monitoring

Genda Chen, Missouri University of Science and Technology (United States)

Haiying Huang, The University of Texas at Arlington (United States)

10A Monitoring Strategies for Bridges

Genda Chen, Missouri University of Science and Technology (United States)

10B Energy Saving and Harvesting Methods for Sensors

Zhu Mao, University of Massachusetts Lowell (United States)

Kenneth A. Cunefare, Georgia Institute of Technology (United States)

11 A Ultrasonic Methods

Wentao Wang, University of Michigan (United States)

Steven Anton, Tennessee Technological University (United States)

$11 \mathrm{~B}$ Control of Large-scale Structures

Hiroshi Asanuma, Chiba University (Japan)

Sung Han Sim, Ulsan National Institute of Science and Technology (Korea, Republic of)

12A Health Assessment of Metallic Structures

Donghyeon Ryu, New Mexico Institute of Mining and Technology (United States)

Salvatore Salamone, The University of Texas at Austin (United States)

12B Novel Sensing Technologies IV

Changgil Lee, Sungkyunkwan University (Korea, Republic of)

13 System Identification and Damage Detection Methods

Fabio Semperlotti, Purdue University (United States) 